\title{
Generalized nets with additional intuitionistic fuzzy conditions for tokens transfer
}

\author{
Dafina Zoteva $^{1}$, Eulalia Szmidt ${ }^{2,3}$ and Janusz Kacprzyk ${ }^{2,3}$ \\ ${ }^{1}$ Department of Bioinformatics and Mathematical Modelling \\ Institute of Biophysics and Biomedical Engineering, Bulgarian Academy of Sciences \\ 105 Acad. Georgi Bonchev Str., Sofia 1113, Bulgaria \\ e-mail: dafy.zoteva@gmail.com \\ ${ }^{2}$ Systems Research Institute, Polish Academy of Sciences \\ ul. Newelska 6, 01-447 Warsaw, Poland \\ ${ }^{3}$ Warsaw School of Information Technology \\ ul. Newelska 6, 01-447 Warsaw, Poland \\ e-mail: szmidt@ibspan.waw.pl, kacprzyk@ibspan.waw.pl
}

\begin{abstract}
A new extension of the concept of generalized nets (GNs), namely GNs with additional intuitionistic fuzzy conditions, is presented in the present paper. The tokens, which are the dynamic components of the net, are assigned with intuitioninstic fuzzy pairs, which are interpreted as degrees of membership and non-membership, and here indicate characteristic of tokens validly passing through a certain route. The new extension is proved to be a conservative extension of the class of the standard GNs, as its functioning and the results of its works can be described by standard GNs.
\end{abstract}

Keywords: Generalized nets, Extensions, Intuitioninstic fuzzy pairs.

2010 Mathematics Subject Classification: 03E72.

\section{Introduction}

Generalized nets (GNs) were introduced in 1982 as an extension of Petri nets and other their extensions and modifications $[2,4,5,8]$. Since then, the concept of GNs itself has been extended 
numerous times. Each of the GN extensions has been proved to be a conservative extension of the standard GNs. In each of the GN extensions, the transfer of tokens between two places is determined by the values of certain predicates. In the case of the standard GNs, these predicates are evaluated as true or false.

Some of the GN extensions combine the concept of GNs with the theory of intuitioninstic fuzzy sets (IFS, [6]). In the case of Intuitionistic Fuzzy GNs of Type 1 (IFGN1) and Intuitionistic Fuzzy GN of Type 2 (IFGN2) the values of the transitions' condition predicates are in the form of Intuitionistic Fuzzy Pairs (IFPs). An IFP [9] is an object $\langle a, b\rangle$, where $a, b \in[0,1]$ and $a+b \leq 1$, which is used as an evaluation of an object or a process, whose components are interpreted as degrees of membership and non-membership, or degrees of validity and non-validity, or degrees of correctness and non-correctness, etc.

The essential difference between IFGN2s and the other extensions of GNs is that the tokens are regarded as "quantities" which do not have initial or other characteristics. Instead of the tokens, in the case of IFGN2, the characteristics are for the places. Intuitionistic fuzzy GN of type 3 (IFGN3) and type 4 (IFGN4) extend the concepts of IFGN1 and IFGN2, respectively. Intuitionistic fuzzy evaluations of the characteristics of the tokens are used in these cases, giving their degrees of truth and falsity, or the validity and non-validity of the characteristics. All these concepts are further extended in [1], adding characteristics of the places with intuitionistic fuzzy estimations, under certain conditions.

Following the ideas of these extensions, a new extension of the concept of the GNs is proposed here, a GN with Additional Intuitionistic Fuzzy Conditions for Tokens Transfer (GNAIFCTT). Each of the tokens is assigned an IFP $\langle\mu, \nu\rangle$, which is later exploit in the assessment of the transitions conditions predicates. It is worth mentioning that the idea of introducing GNAIFCTT is motivated both from practical and theoretical points of view. In the real world problems with imprecise and hesitant knowledge prevail so having a possibility to take it into account for tokens of GNs seems natural. On the other hand, the problem is also interesting theoretically, as it will be shown in the next sections.

The paper is organized as follows: the formal definitions related to the GNAIFCTT are presented in Section 2. The proof that the new extension is a conservative one with respect to the class of the standard GNs is provided in Section 3. The concluding remarks are given in Section 4.

\section{Definitions}

A GN of each type (a standard GN or a conservative GN extension) contains transitions, places and tokens. The new extension of GNs requires a slight modification of the definition of a GNtransition. The differences from the general definition of a GN-transition will be shown before the formal definition of a GNAIFCTT. 


\subsection{Transition in a Generalized net with additional intuitionistic fuzzy conditions for tokens transfer}

Every transition in a GN with additional IF conditions for token transfer is defined by a seventuple:

$$
Z_{A I F C}=\left\langle L^{\prime}, L^{\prime \prime}, t_{1}, t_{2}, \bar{r}, C, \square\right\rangle
$$

where

(a) $L^{\prime}$ and $L^{\prime \prime}$ are finite, non-empty sets of places - the transition's input and output places, respectively. $L^{\prime}=\left\{l_{1}^{\prime}, \ldots, l_{i}^{\prime}, \ldots, l_{m}^{\prime}\right\}, L^{\prime \prime}=\left\{l_{1}^{\prime \prime}, \ldots, l_{j}^{\prime \prime}, \ldots, l_{n}^{\prime \prime}\right\}$.

(b) $t_{1}$ is the current time-moment of the transition's firing;

(c) $t_{2}$ is the current value of the duration of its active state;

(d) $\bar{r}$ is an index matrix (IM) [3, 7] with the conditions for transfer of certain tokens from the transition's input places to corresponding outputs. The IM $\bar{r}$ has the following form:

$$
\begin{array}{c|c} 
& l_{1}^{\prime \prime} \ldots l_{j}^{\prime \prime} \ldots l_{n}^{\prime \prime} \\
\hline l_{1}^{\prime} & \\
\vdots & \bar{r}_{i, j}=\left\langle r_{i, j}, M, N\right\rangle \\
l_{i}^{\prime} & \left(r_{i, j}-\text { predicates }\right) \\
\vdots & (1 \leq i \leq m, 1 \leq j \leq n) \\
l_{m}^{\prime} &
\end{array}
$$

where the element $r_{i, j}$ is a predicate in terms of the definition of the standard GNs, which can be evaluated as true or false, while $M, N \subseteq[0,1]$. The transfer of a token $\alpha$ from the $i^{\text {th }}$ input to the $j^{\text {th }}$ output place under the additional IF condition $\left\langle\mu_{\alpha}, \nu_{\alpha}\right\rangle$, assigned to the token $\alpha$, is determined by the function $f$ of the GNAIFCTT definition (Section 2.2). It is only possible when $f\left(\bar{r}_{i, j}\right)$ is true;

(e) $C$ is an IM of the capacities of the transition's arcs:

$$
C=\begin{array}{c|c} 
& l_{1}^{\prime \prime} \ldots l_{j}^{\prime \prime} \ldots l_{n}^{\prime \prime} \\
\hline l_{1}^{\prime} & \\
\vdots & c_{i, j} \\
l_{i}^{\prime} & \left(c_{i, j} \in \mathcal{N},\right. \\
\vdots & 1 \leq i \leq m, 1 \leq j \leq n) \\
l_{m}^{\prime} &
\end{array},
$$

where $\mathcal{N}=\{0,1,2, \ldots\} \cup\{\infty\}$.

(f) $\square$ is the transition's type. It has the form of a Boolean expression with the identifiers of the transition's input places as variables, and the Boolean operations $\wedge$ and $\vee$. This formula has the following semantics: 
$\wedge\left(l_{i_{1}}, l_{i_{2}}, \ldots, l_{i_{u}}\right)$ - there has to be at least one token in each of the places $l_{i_{1}}, l_{i_{2}}, \ldots, l_{i_{u}}$, $\vee\left(l_{i_{1}}, l_{i_{2}}, \ldots, l_{i_{u}}\right)$ - there has to be at least one token in one of all places $l_{i_{1}}, l_{i_{2}}, \ldots, l_{i_{u}}$, where $\left\{l_{i_{1}}, l_{i_{2}}, \ldots, l_{i_{u}}\right\} \subset L^{\prime}$.

The transition can become active only when the value of the transition's type, evaluated as a Boolean expression, is true.

\subsection{Definition of a generalized net with additional intuitionistic fuzzy conditions for tokens transfer}

The ordered four-tuple

$$
E_{A I F C}=\left\langle\left\langle A, \pi_{A}, \pi_{L}, c, f, \theta_{1}, \theta_{2}\right\rangle,\left\langle K_{I F V T}, \pi_{K}, \theta_{K}, V_{I F V T}\right\rangle,\left\langle T, t^{o}, t^{*}\right\rangle,\langle X, \Phi, b\rangle\right\rangle
$$

is called a Generalized Net with Additional Intuitionistic Fuzzy Conditions for Tokens Transfer (GNAIFCTT), if:

(a) $A$ is a set of transitions, defined according to the definition in Section 2.1;

(b) $\pi_{A}$ is a function which gives the priorities of the transitions, i.e., $\pi_{A}: A \rightarrow \mathcal{N}$;

(c) $\pi_{L}$ is a function which gives the priorities of the places, i.e., $\pi_{L}: L \rightarrow \mathcal{N}$, where $L=$ $p r_{1} A \cup p r_{2} A$, i.e., $L$ is the set of all GN-places, and $\operatorname{pr}_{i}\left\{x_{1}, x_{2}, \ldots, x_{n}\right\}=x_{i}$, where $n \in \mathcal{N}$, $n \geq 1$ and $1 \leq k \leq n$

(d) $c$ is a function which gives the capacities of the places, i.e., $c: L \rightarrow \mathcal{N}$;

(e) $f$ is a function which evaluates the truth values of the predicates of the transition's conditions. Despite the more complex nature of the elements in the IM $r$ in each transition, the values obtained by this function are true and false, just like the case of the standard GNs. Again, if $\bar{r}_{i j}=\left\langle r_{i j}, M, N\right\rangle$ is the predicate in the transition conditions, which should determine the transfer from the $i^{t h}$ input to the $j^{t h}$ output place under the additional IF condition $\left\langle\mu_{\alpha}, \nu_{\alpha}\right\rangle$, assigned to the token $\alpha$, then $f\left(\bar{r}_{i, j}\right)$ is evaluated as

$$
f\left(\bar{r}_{i, j}\right)=f\left(r_{i, j}\right) \cdot f\left(" \mu_{\alpha} \in M "\right) \cdot f\left(" \nu_{\alpha} \in N "\right) .
$$

This transfer is only possible when $f\left(\bar{r}_{i, j}\right)$ is true.

Since the additional IF conditions $\langle\mu, \nu\rangle$ are assigned to each token at the beginning of the GN functioning and they remain constant during the GN functioning, an answer could be given to a question like "Is there a chance that the token will pass through all the places of a certain route?".

Let $\left\{\bar{r}_{i j}=\left\langle r_{i j}, M, N\right\rangle\right\}$ be the set of all predicates which need to be estimated in a given route. If $\mu \notin[\max \inf M, \min \sup M]$ or $\nu \notin[\max \inf N, \min \sup N]$, then this route is not possible for the token and there is no need for the predicates $r_{i j}$ to be evaluated. 
(f) $\theta_{1}$ is a function which gives the next moment of time when a transition can be activated, i.e., $\theta_{1}(t)=t^{\prime}$, where $t=p r_{3} Z$, and $t, t^{\prime} \in\left[T, T+t^{*}\right]$ and $t \leq t^{\prime}$. The value of this function is calculated when the transition terminates its active state;

(g) $\theta_{2}$ is a function which gives the duration of the active state of a transition, i.e., $\theta_{2}(t)=t^{\prime}$, where $t=\operatorname{pr}_{4} Z, t \in\left[T, T+t^{*}\right]$ and $t^{\prime} \geq 0$. The value of this function is calculated at the moment of the transition's activation;

(h) $K_{A I F C}$ (or $K$ ) is the set of tokens to which an additional IF condition will be assigned, in order to determine the possibility for their transfer;

(i) $\pi_{K}$ is a function which gives the priorities of the tokens, i.e., $\pi_{K}: K \rightarrow \mathcal{N}$;

(j) $\theta_{K}$ is a function which gives the moment of time when a certain token can enter the net, i.e., $\theta_{K}(\alpha)=t$, where $\alpha \in K$ and $t \in\left[T, T+t^{*}\right]$;

(k) $V_{A I F C}$ is the function which assigns the additional IF conditions to each token upon entering the net. For now, this condition will not be changed during the functioning of the GNAIFCTT. $V_{A I F C}: K \rightarrow\{\langle a, b\rangle \mid a, b \in[0,1], a+b \leq 1\}$;

(1) $T$ is the moment of time when the GN starts functioning. This moment is determined with respect to a fixed (global) time-scale;

(m) $t^{o}$ is an elementary time-step related to the fixed (global) time-scale;

(n) $t^{*}$ is the duration of the GN functioning;

(o) $X$ is the function which assigns initial characteristics to each of the tokens when they enter the GN;

(p) $\Phi$ is a characteristic function which gives a new characteristic to each token upon its transfer from an input to an output place of a certain transition;

(q) $b$ is a function which gives the maximum number of characteristics a certain token can receive, i.e., $b: K \rightarrow \mathcal{N}$.

\subsection{Algorithm for transition functioning}

The general algorithm for transition's functioning, described in $[4,5,8]$, is slightly modified here to take into consideration the specifics of the newly defined GNAIFCTT. The algorithm for tokens transfer after the moment of time $t_{1}=$ time (the current GN moment of time), denoted by algorithm $A$, takes into consideration the possibilities of merging and splitting tokens.

The list of tokens which can be merged with a given token $\alpha$ have to be specified in the initial characteristics of this $\alpha$ token. For example, the expression

$$
x_{0}^{\alpha}="\left\langle\left\{\beta_{1}, \ldots, \beta_{k}\right\}, x_{0}^{\alpha, *}\right\rangle "
$$


denotes that the $\alpha$ token can be merged with the tokens in the set $\left\{\beta_{1}, \ldots, \beta_{k}\right\}$. The rest of the information of the token's initial characteristics is stored in $x_{0}^{\alpha, *}$.

The algorithm A can be described in 12 steps, as follows:

A01 Sort the input and output places of the transitions by their priorities.

A02 Form two lists of tokens in each input place $l$. The first list contains those of the tokens that might be transferred to a certain output place during the current time moment. Sort these tokens by their priorities. The second list is empty at first. The two lists shall be denoted with $P_{1}$ and $P_{2}$, respectively.

A03 Generate an empty index matrix $R$ that corresponds to the index matrix of the predicates $r$. The values in the matrix $R$ can only be 0 or 1 , which correspond to predicate evaluations false and true. Assign 0 to all elements $R_{i, j}$ of $R$ which:

- are in a row that corresponds to an empty input place, i.e., there are no tokens in the input place that can be transferred to an output place of the current transition;

- are placed in the position $(i, j)$ for which the predicate $r_{i, j}$ is set as false or $c_{i, j}=0$, i.e., the current capacity of the arc between the $i^{\text {th }}$ input place and the $j^{t h}$ output place is 0 .

Proceed with step A04.

A04 Iterate through the input places in the order set by their priorities, starting with the place with highest priority for which no token has been transferred during the current time-step and which has at least one token in it. The token $\alpha$, which might be transferred on the current time-step with the additional IF condition $\left\langle\mu_{\alpha}, \nu_{\alpha}\right\rangle$, is the one with the highest priority in the $P_{1}$ list of the current input place. Perform the following steps in order to determine if and where to transfer the current token.

A04a Find the next $R_{i, j}$ value on the relevant row of the IM $R$, which has not been set on the current time-step. If such a value exists, go to step A04b. If all the values on the relevant row of the IM $R$ have been checked, go to step A04e.

A04b Check if the relevant output place, which the token $\alpha$ might be transferred to, is full. If so, go to step A04c, otherwise go to step A04d.

A04c Check if the relevant output place has a token (or tokens) that can be merged with the one being transferred. If so, go to step A04c. Otherwise, go to step A04d.

A04d Evaluate the truth value of the corresponding predicate $\bar{r}_{i, j}$ of the index matrix $r$. If $f\left(\bar{r}_{i, j}\right)=$ true, set the $R_{i, j}$ value of $R$ to 1 and proceed with step A04e. Otherwise, set the $R_{i, j}$ value to 0 and go to step A04a.

A04e If all the values on the relevant row are 0, go to step A05. Otherwise, go to step A04f. 
A04f If there are $R_{i, j}$ values set to 1 , the token $\alpha$ splits in as many new tokens as the number of the $R_{i, j}$ values set to 1 . These newly generated tokens are identical to the original token $\alpha$. They are transferred to the corresponding output places under the same additional IF conditions $\left\langle\mu_{\alpha}, \nu_{\alpha}\right\rangle$ and are merged with specified tokens in the new hosts, if there are such.

Since these additional conditions $\langle\mu, \nu\rangle$ could be considered as a degree of shrinking within certain limits, or a degree of passing through a certain route, when the token $\alpha$ is merged with a token $\beta$ in a relevant output place, the following strategy for merging could be considered, the additional IF condition for the token $\beta$ to be changed to $\left\langle\min \left(\mu_{\alpha}, \mu_{\beta}\right), \max \left(\nu_{\alpha}, \nu_{\beta}\right)\right\rangle$.

Evaluate the characteristic function of these output places. Assign these values as new characteristics to the corresponding transferred tokens upon entering the output places.

Proceed with step A05.

A05 If the highest priority token cannot be transferred during the current time step, move the token to the $P_{2}$ list of the input place.

A06 Increase by 1 the current number of tokens in each output place to which a token has been transferred if the token has not been merged with any of the other tokens in the host. Do not change the current number of tokens in the output place otherwise.

A07 Decrease by 1 the current number of tokens in each input place from which a token has been transferred. If the current number of tokens in such an input place becomes 0 , set to 0 all the elements in the corresponding row of the index matrix $R$.

A08 Decrease by 1 the capacities of all the arc through which a token has been transferred. If the current capacity of an arc becomes 0 , assign 0 to this element of the index matrix $R$ that corresponds to the arc.

A09 If there are more input places with lower priority from which no token has been transferred to an output place, go to step A04. Otherwise, go to step A10.

A10 Add $t^{0}$ to the current model time.

A11 If the value of the current time is less than or equals $t_{1}+t_{2}$ (the time components of the considered transition), go to A04. Otherwise, go to step A12.

A12 End of the transition's functioning.

The general algorithm for GNAIFCTT functioning is no different from the one for the standard GN. 


\section{GNAIFCTT as a conservative extension of the standard generalized net}

Let $\Sigma$ be the class of the standard GNs and $\Sigma_{A I F C}$ be the class of the GNAIFCTT. Every standard GN is a GNAIFCTT, i.e., the relation

$$
\Sigma \vdash \Sigma_{A I F C}
$$

holds.

Every standard GN can be seen as a GNAIFCTT in which $V_{A I F C}(\alpha)=\langle 1,0\rangle, \forall \alpha \in K$ and the intervals $M, N$ are $[0,1], \forall \bar{r}_{i, j}$ in the IM $\bar{r}$ with the transitions' condition predicates.

Therefore, GNAIFCTTs are extensions of the standard GNs.

In addition, every GNAIFCTT can be represented as a standard GN, i.e., GNAIFCTTs are conservative extensions of GNs.

Theorem 1. The class $\Sigma_{A I F C}$ is a conservative extension of the class $\Sigma$, i.e.,

$$
\Sigma_{A I F C} \equiv \Sigma .
$$

Proof. It is sufficient to show that the functioning and the results of the work of every GNAIFCTT can be described by some ordinary GN.

Let the GNAIFCTT $E$ be given

$$
E=\left\langle\left\langle A, \pi_{A}, \pi_{L}, c, f, \theta_{1}, \theta_{2}\right\rangle,\left\langle K_{A I F C}, \pi_{K}, \theta_{K}, V_{A I F C}\right\rangle,\left\langle T, t^{o}, t^{*}\right\rangle,\langle X, \Phi, b\rangle\right\rangle .
$$

A standard GN $G$ will be constructed, with the same time components and the same number of transitions as $E$. For every transition $Z$ in $E$, a corresponding transition $Z^{G}$ in $G$ will be constructed so that the tokens which enter the corresponding input places with the same characteristics are transferred to the corresponding output places with equal characteristics.

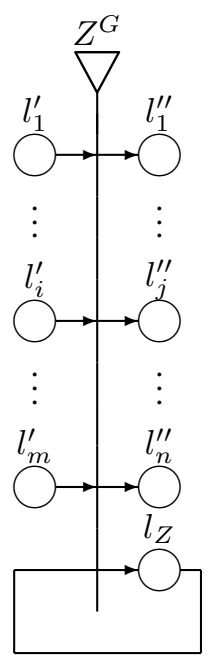

Figure 1. $Z^{G}$ of $G$ 
Let $G$ be a standard GN with the following definition:

$$
G=\left\langle\left\langle A^{G}, \pi_{A}, \pi_{L}^{G}, c, f^{G}, \theta_{1}, \theta_{2}\right\rangle,\left\langle K^{G}, \pi_{K}^{G}, \theta_{K}^{G}\right\rangle,\left\langle T, t^{o}, t^{*}\right\rangle,\left\langle X^{G}, \Phi^{G}, b\right\rangle\right\rangle
$$

$A^{G}$ is the set of transitions of $\mathrm{G}$. Let $Z^{E}$ be an arbitrary transition in the GNAIFCTT $E$

$$
Z^{E}=\left\langle L^{\prime}, L^{\prime \prime}, t_{1}, t_{2}, \bar{r}, C, \square\right\rangle
$$

The corresponding transition in the standard GN $G$ will be defined in the following way (see Fig. 1)

$$
Z^{G}=\left\langle L_{G}^{\prime}, L_{G}^{\prime \prime}, t_{1}, t_{2}, r^{G}, M^{G}, \square^{G}\right\rangle
$$

where $L_{G}^{\prime}=L^{\prime} \cup\left\{l_{Z}\right\}, L_{G}^{\prime \prime}=L^{\prime \prime} \cup\left\{l_{Z}\right\}, \square^{G}=\wedge\left(\square, l_{Z}\right)$.

An additional place $l_{Z}$ is added to every transition $Z$ of $E$ with a token $\omega_{Z}$, which enters the net with the start of its functioning and stays in $l_{Z}$ throughout the whole GN functioning. The initial characteristic of this token is the set of intervals $\left\{\left(M_{i, j}, N_{i, j}\right) \mid i \in p r_{1} Z^{G}, j \in p r_{2} Z^{G}\right\}$ used in the IM $\bar{r}$ in the definition of $Z^{E}$. The place $l_{Z}$ has the highest priority among the places of the transition $Z^{G}$, so that the estimation of the predicates could be possible.

If $\bar{r}=p r_{5} Z^{E}=\left[L^{\prime}, L^{\prime \prime},\left\{\bar{r}_{l_{i}, l_{j}}\right\}\right]$ has the form of an IM, them the corresponding IM $r^{G}$ has the form:

$$
\begin{gathered}
r^{G}=p r_{5} Z^{G}=\left[L^{\prime} \cup\left\{l_{Z}\right\}, L^{\prime \prime} \cup\left\{l_{Z}\right\},\left\{r_{l_{i}, l_{j}}^{G}\right\}\right], \\
\left(\forall l_{i} \in L^{\prime}\right)\left(\forall l_{j} \in L^{\prime \prime}\right)\left(r_{l_{i}, l_{j}}^{G}=\bar{r}_{l_{i}, l_{j}}\right), \\
\left(\forall l_{i} \in L^{\prime}\right)\left(\forall l_{j} \in L^{\prime \prime}\right)\left(r_{l_{i}, l_{Z}}^{G}=r_{l_{Z}, l_{j}}^{G}=\text { false }\right), \\
r_{l_{Z}, l_{Z}}^{G}=\text { true. }
\end{gathered}
$$

If $C=\operatorname{pr}_{6} Z^{E}=\left[L^{\prime}, L^{\prime \prime},\left\{c_{l_{i}, l_{j}}\right\}\right]$ has the form of an IM, them the corresponding IM $M^{G}$ has the form:

$$
\begin{gathered}
M^{G}=p r_{6} Z^{G}=\left[L^{\prime} \cup\left\{l_{Z}\right\}, L^{\prime \prime} \cup\left\{l_{Z}\right\},\left\{m_{l_{i}, l_{j}}^{G}\right\}\right], \\
\left(\forall l_{i} \in L^{\prime}\right)\left(\forall l_{j} \in L^{\prime \prime}\right)\left(m_{l_{i}, l_{j}}^{G}=c_{l_{i}, l_{j}}\right), \\
\left(\forall l_{i} \in L^{\prime}\right)\left(\forall l_{j} \in L^{\prime \prime}\right)\left(m_{l_{i}, l_{Z}}^{G}=m_{l_{Z}, l_{j}}^{G}=0\right), \\
m_{l_{Z}, l_{Z}}^{G}=1 .
\end{gathered}
$$

$A^{G}$ is the set of all transitions of the standard GN $G$, constructed based on the transitions of the GNAIFCTT $E$.

$\pi_{L}^{G}=\pi_{L} \cup \pi_{\left\{l_{Z} \mid Z \in A\right\}}$, where the function $\pi_{\left\{l_{Z} \mid Z \in A\right\}}$ determines the priorities of the additional places $l_{Z}$ as the highest among the priorities of the places of the transition $Z \in A$.

$c^{G}=c \cup c_{\left\{l_{Z} \mid Z \in A\right\}}$, where $c_{\left\{l_{Z} \mid Z \in A\right\}}\left(l_{Z}\right)=1$.

$K^{G}=K_{A I F C} \cup\left\{\omega_{Z} \mid Z \in A\right\}$, i.e., the set of tokens of the ordinary GN includes all the tokens of the GNAIFCTT $E$ and the additional tokens $\omega_{Z}$ in the $l_{Z}$ places.

The function $X^{G}$ which determines the initial characteristics of the tokens is defined as $X^{G}=X \cup\left\{x_{0}^{\alpha} \mid \alpha \in K, x_{0}^{\alpha}=V_{A I F C}(\alpha), x_{0}^{\alpha} \in\{\langle a, b\rangle \mid a, b \in[0,1], a+b \leq 1\}\right\} \cup\left\{x_{0}^{\omega_{Z}} \mid Z \in A\right\}$. 
$\Phi^{G}=\Phi \cup \Phi_{\left\{\omega_{Z} \mid Z \in A\right\}}$, where $\Phi_{\left\{\omega_{Z} \mid Z \in A\right\}}\left(\omega_{Z}\right)=\varnothing$, i.e., no additional characteristics are assigned to the additional tokens $\omega_{Z}$ in each transition $Z^{G}$.

Now, do the two GNs $E$ and $G$ function equally?

The functioning and the results of the work of the transition $Z^{G}$ in $G$ will be compared to the functioning and, respectively, the results of the work of its corresponding transition $Z^{E}$ in $E$.

These two transitions become active at the same moment of time and have equal duration of the functioning. The priorities and the capacities of the corresponding places are equal, as well as the capacities of the arcs.

Two arbitrary tokens, $\alpha^{E}$ and its corresponding token $\alpha^{G}$, different from the additional tokens $\omega_{Z}$ in the places $l_{Z}$, will enter the two corresponding input places of the transitions $Z^{E}$ and $Z^{G}$ at the same time. The token $\alpha^{G}$ will have all the initial characteristics of $\alpha^{E}$, plus the additional IF condition $\left\langle\mu_{\alpha^{G}}, \nu_{\alpha^{G}}\right\rangle$ for the transfer. The set of intervals $M_{l_{i}, l_{j}}$ and $N_{l_{i}, l_{j}}$, necessary for the evaluations of the transition conditions predicates, could be found in the initial characteristics of the token $\omega_{Z}$. The predicated in both transitions conditions $\left(Z^{E}\right.$ and $\left.Z^{G}\right)$, therefore, will be the evaluated as equal.

The two tokens will be transferred to corresponding output places and will receive the same characteristics. The characteristic functions in both of the nets are the same for all the corresponding places. The only difference in $G$ is that no additional characteristics are assigned to the tokens $\omega_{Z}$ in the additional places of the transitions $Z^{G}$.

Therefore, the corresponding tokens behave in the same way. Since they are chosen randomly, it can be concluded that the same assertion can be made for any other pair of corresponding tokens of the nets. This leads to the point that the two corresponding transitions $Z^{E}$ and $Z^{G}$ have identical behaviour. Hence, the standard GN $G$ can describe the functioning and the work of the GNAIFCTT $E$.

\section{Conclusion}

A new extension of the concept of the standard generalized nets is presented here with additional intuitionistic fuzzy conditions for the transfer of each token. The possibilities of its application will be studied in the near future. One feasible direction of further studies is the construction of IF histograms [10] at each time-step of the GN's functioning. The IF modal operators "necessity" and "possibility" could be applied on the IF histograms in order to determine to what extend the places of the net have been filled.

In addition, all the possible routes for a certain token $\alpha$ could be estimated. Since the additional IF conditions do not change during the GN functioning, an answer could be given to a question like "Is there a chance that the token will pass through all the places of a certain route?". If $\langle\mu, \nu\rangle$ for each token are to be changed during the GN functioning, this kind of task will become undetermined. This will be a matter of further research. 


\section{Acknowledgements}

This work is partially supported by the National Science Fund of Bulgaria under Grant DN02/10 "New Instruments for Knowledge Discovery from Data, and their Modelling."

\section{References}

[1] Andonov, V. (2013). Intuitionistic fuzzy generalized nets with characteristics of the places of type 1 and type 3. Notes Intuitionistic Fuzzy Sets, 19 (3), 99-110.

[2] Atanassov, K. (1985). The Generalized nets and the other graphical means for modelling. AMSE Review, 2 (1), 59-64.

[3] Atanassov, K. (1987). Generalized index matrices. Comptes rendus de l'Academie Bulgare des Sciences, 40 (11), 15-18.

[4] Atanassov, K. (1991). Generalized Nets. Singapore, World Scientific.

[5] Atanassov, K. (2007). On Generalized Nets Theory'. "Prof. M. Drinov" Academic Publishing House, Sofia.

[6] Atanassov, K. (2012). On Intuitionistic Fuzzy Sets Theory. Springer, Berlin.

[7] Atanassov, K. (2014). Index Matrices: Towards an Augmented Matrix Calculus. Springer, Cham.

[8] Atanassov, K. \& Sotirova, E. (2017). Generalized Nets Theory. "Prof. M. Drinov" Academic Publishing House, Sofia (in Bulgarian).

[9] Atanassov, K., Szmidt, E. \& Kacprzyk, J. (2013). On intuitionistic fuzzy pairs. Notes on Intuitionistic Fuzzy Sets, 19 (3), 1-12.

[10] Atanassova, L., Gluhchev, G. \& Atanassov, K. (2010). On intuitionistic fuzzy histograms. Notes in Intuitionistic Fuzzy Sets, 16 (4), 31-36. 